\title{
Editorial
}

\section{Como avanzar en consenso hacia las modalidades de parto y nacimiento integral}

\author{
Claudia Uribe T, PhD, MSc, EM
}

Profesor Asociado, Pontificia Universidad Católica de Chile

El camino hacia la recuperación de un proceso de parto y nacimiento más personalizado e integral ha tomado, en Chile, alrededor de dos décadas. Esto puede entenderse, producto de la inercia generada en el sistema satisfecho con los logros en materia de seguridad en la maternidad en los últimos treinta años. De este modo, podría asumirse que los indicadores maternos y perinatales alcanzados se deban a la alta intervención a los que han estado expuestos los nacimientos en este periodo, más que al resultado de un sistema consolidado de alta calidad en la vigilancia prenatal y de la asistencia profesional del parto. Respecto a esto último se invita al lector a observar desde su participación y su rol en el proceso, cómo pudiese transformarse el nacimiento, en Chile, en una experiencia positiva (1), además de segura.

La reflexión de la comunidad profesional, en torno a la implementación de una política de intervención del proceso de parto integral, no ha estado exenta de discrepancias. Lo que hasta el día de hoy prevalece como modalidad estándar corresponde a un modelo más intervencionista, el cual, arraigado en el tiempo, se transmite de generación en generación en las disciplinas y profesiones relacionadas con el parto y nacimiento, no atribuible exclusivamente al ámbito médico.

El profesional y el equipo en general, responsable de la asistencia y cuidado en el proceso de parto, pudiese estar formado y preparado para otorgar una atención más intervenida, de manera generalizada; o capacitado para discernir en cada situación particular respecto de las intervenciones necesarias y de la utilización de tecnología y procedimientos invasivos debidamente justificados.

Cabe destacar, que cuando nos enfrentamos a situaciones de alto riesgo obstétrico y perinatal los beneficios de la medicalización y de las medidas de conducción del parto, así como la resolución por cesárea son innegables desde el punto de vista de la seguridad del proceso. Si ponemos atención en los indicadores nacionales en materia de seguridad materna y neonatal, es conocido que en las últimas décadas nos hemos mantenido con cifras similares a los países desarrollados. No obstante, dichos países también han mejorado en términos de la calidad del cuidado. Los avances van en la línea de la implementación de modelos o modalidades "humanizadas", "personalizadas" o "integrales" que dan visibilidad del nacimiento, como un suceso fisiológico y de naturaleza social-afectiva, al que sólo se guía, se acompaña, y al cual se interviene y se conduce justificadamente, si presenta riesgo o se complica. En ambos casos, la asistencia y cuidado debe considerar la comprensión de los factores psicosociales en torno al evento. Hoy estamos convocados a materializar los nuevos desafíos para hacer del nacimiento una experiencia positiva para la mujer, su hijo y familia. No podemos desconocer que existen recomendaciones claras y explícitas desde hace más de tres décadas de parte de la Organización Mundial de la Salud (OMS)(2). En Fortaleza, Brasil la OMS alertó en su Declaración sobre la Apropiada Tecnología del Nacimiento, destacando la importancia del rol protagónico de la mujer en el planeamiento y desarrollo de su proceso; la participación familiar (en ese entonces, una persona significativa); la promoción de técnicas de vinculación con su hijo(a) y la debida intervención, ajustada a los requerimientos de la progresión del parto. Para entonces, ya se disponía de evidencia científica que demostraba el potencial desvío de la normalidad en aquellos procesos de parto, que aun siendo de bajo riesgo, se exponen a 
una plantilla de conducción sin discriminar entre lo debidamente justificado y lo normalmente establecido. ¿Cuál sería el fundamento, si existe evidencia suficiente que avala las prácticas de conducción sólo en casos necesarios, y que promueve fuertemente que el parto, en un entorno social y afectivo, es positivo para la mujer, su recién nacido y familia?

En Chile este movimiento y el cambio de paradigma ha sido aún más lento. Es posible que las primeras propuestas que surgieron en Chile estaban basadas en una fuerte crítica hacia las atenciones relacionadas al modelo biomédico y consideradas como prácticas "deshumanizadas", creando con ello un marco de resistencia natural de parte del sistema. Por otra parte, muchas de las iniciativas se han basado en un trabajo riguroso de capacitación con énfasis en las usuarias. Con esto último, podría lograrse que la población de usuarias y familias se empoderen para vivir una experiencia especial del nacimiento, y se encuentren en el momento con un sistema de atención sin preparación previa y resistentes a los procesos, porque el equipo de salud no ha sido debidamente integrado. Pareciera lógico pensar, que un cambio de paradigma de tal magnitud debe ir de la mano de una "intervención más compleja", donde todos los actores, tanto del sistema de salud como los usuarios y el entorno social, participen colaborativamente del cambio.

En Chile se comenzó con experiencias pilotos aisladas, desde la década de los 90 , siendo escasas las que fueron documentadas y más aún, casi nulas, las que aportaron al conocimiento de manera rigurosa. Por todos es sabido que sin ciencia y evidencia es difícil recomendar y sensibilizar.

A partir de la primera década del milenio se dan a conocer nuevas experiencias y se generan de manera sistemática iniciativas relacionadas al bienestar materno y parto personalizado a nivel país (3), conducentes a la implementación de modalidades integrales basadas en la calidad y seguridad (4).

No obstante, los logros alcanzados a la fecha, nos encontramos en el inicio del camino, y mucho por hacer en el presente y futuro. Las mujeres que han vivido experiencias negativas de parto han tomado la delantera y se han pronunciado. Por qué esperar que emerjan proyectos de ley que regulen la asistencia y el cuidado en el nacimiento, si en cada uno de nosotros, los profesionales, existe una motivación intrínseca para promover experiencias positivas.

La invitación para toda la comunidad profesional y científica que se relaciona con el maravilloso evento de la llegada al mundo (vivido por todos nosotros) es, ante todo, al trabajo participativo con la usuaria-familia y a iniciar un proceso de toma de decisiones compartida; a la implementación interdisciplinaria de propuestas pilotos; a validar los procedimientos integrativos y complementarios a la intervención estándar; y a construir conocimiento sólido respecto de las experiencias de modalidades integrales ya implementadas. A modo de ejemplo, destacar las iniciativas de investigación y expresión de difusión del conocimiento, como lo es el artículo del presente número, que revela la experiencia de mujeres que vivieron su proceso de parto integral en el ámbito público y privado.

La intención de la presente es motivar a la comunidad de especialistas para integrar y co-diseñar las estrategias de intervención presentes y futuras para hacer de la experiencia del parto una experiencia positiva y compartida, logrando así, mejores nacimientos para Chile.

\section{REFERENCIAS}

1. World Health Organization. WHO recommendations: Intrapartum care for a positive childbirth experience [Internet]. Green Ink. UK; 2018. 212 p. Available from: http://apps.who.int/iris/bitstream/10665/260178/1/ 9789241550215-

eng.pdf?ua=1\%0Ahttp://www.who.int/reproductive health/publications/intrapartum-care-

guidelines/en/

2. WHO. Appropriate technology of birth. Lancet. 1985;2(8452):436-7.

3. Binfa L, Pantoja L, Ortiz J, Gurovich M, Cavada G. Assessment of the implementation of the model of integrated and humanised midwifery health services in Santiago, Chile. Midwifery [Internet]. 2013;29(10):1151-7. Available from: http://dx.doi.org/10.1016/j.midw.2013.07.001

4. Uribe C, Contreras A, Bravo P, Villarroel L, Abarzúa F. Modelo de asistencia integral del parto: Concepto de integralidad basado en la calidad y seguridad . Rev Chil Obs Ginecol. 2018;83(3):266-76. the Women's Health Initiative randomized controlled trial. JAMA. 2002 Jul 17;288(3):321-33. 